\title{
Direct Contagion in Financial Networks with Mark-to-Market and Historical Cost Accounting Rules
}

\author{
Mario Eboli \\ Facoltà di Economia, Università "G. d'Annunzio" \\ viale Pindaro 42, 65127 Pescara, Italy
}

Tel: 39-085-453-7582 E-mail: m.eboli@unich.it

I thank Leonardo Cangelmi, Riccardo Palumbo and Paolo Vitale for helpful discussions and suggestions. I also thank the participants of the 6th Applied Financial Economics Conference, held in Samos (Greece) in July 2009, and of the First International Symposium in Computational Economics and Finance, held in Sousse, February 2010, for their useful comments. As customary, I am the sole responsible for any shortcoming that can be found in these pages.

\begin{abstract}
In this paper we compare the effects of two accounting rules, the mark-to-market and the historical cost regimes, on the dynamics of direct, balance sheet contagion in financial networks. This is done using a flow-network representation of a financial system and of the propagation of losses that crosses it as a consequence of a negative shock. We show that, for any network and any shock, the flow of losses generated with the mark-to-market rule is larger than the one generated by accounting at historical cost. This implies that a financial network is more exposed to default contagion, both in terms of scope and threshold of contagion, under the marking-to-market accounting regime, than with the historical cost regime.
\end{abstract}

Keywords: Contagion, Financial networks, Systemic risk, Mark-to-market

JEL classification: C63, G01, G33.

\section{Introduction}

Since the start of the current subprime mortgage crisis, the mark-to-market accounting rule (known also as fair value rule) has come under scrutiny for its alleged role in exacerbating the magnitude and diffusion of financial distress. As is known, such a rule requires that the marketable assets held by a company, that are not classified as 'held-to-maturity', have to be accounted for at market value.(Note 1) Some economists studied the effects on financial contagion of this accounting practice before the subprime crises put it under scrutiny. Shin et al. (2005) highlighted the detrimental interaction between this accounting rule and the loss of value of illiquid assets due to fire sales. When liquidity shortages and/or capital requirements force some agents to sell illiquid assets, the market value of such assets diminishes. This loss of value is borne by all agents who hold those assets, losses which are accounted for in the marked-to-market headings of their balance sheets. These 'marking-to-market'- losses can generate further contagion in as much as they induce further fire sales (possibly also due to the liquidation of defaulting agents) and further reductions of the market value of illiquid assets. The transmission of losses due to the marking-to-market of assets is also studied by Allen and Carletti (2008). These authors show that, with mark-to-market accounting and when the price of illiquid assets depends on market liquidity, a liquidity shortage can generate contagion between a banking sector and an insurance sector. In that paper, as well as in Shin et al. (2005), the channel of contagion is the marking-to-market of price variations of common or correlated assets. In the present paper we analyze the effects of marking-to-market on a different channel of contagion, namely the direct balance-sheet transmission of losses. In networks of financially interconnected agents, where someone's assets are someone else's liabilities, the marking-to-market of such assets creates the grounds for a domino effect of debt deflation that occurs even in the absence of defaults. When a financial operator suffers a loss of value of some of its assets, this loss diminishes the market value of its outstanding debt. Then the holders of such debt, in as much as they mark it to market, suffer a loss that, in turn, worsens their own balance sheets and shrinks the market value of their own outstanding debt. In turn, this loss is borne by the subscribers of such debt, and so forth and so on along the chains of financial obligations that constitutes the fabric of the system, diffusing and amplifying the initial negative shock.

To shed some light on this form of contagion, we use the graph-theoretic representation of financial networks put forward by Eboli (2010b), where a financial system is modeled as a flow-network, and contagion is modeled as a flow of losses that crosses such a network. Building on this framework, we model the propagation of losses, generated by an exogenous shock, under the historical cost and the mark-to-market accounting rules. We show that, for any network and any shock that perturbates it, the flow of losses caused by a shock is larger with the 
marking-to-market rule than with the historical cost rule. As a consequence, we establish that financial networks are more exposed to direct default contagion with the mark-to-market regime than with the historical cost regime, both in terms of contagion scope (the number of defaults) and of contagion threshold, i.e., the value of the smallest shock that is large enough to cause default contagion.

The paper is organized as follows: Section two presents the flow network representation of a financial system while section three presents the two different propagations of losses - with mark-to-market rule and historical cost rule, respectively - which are induced, in such a network, by an exogenous shock. In section four, these two propagations are compared with respect to the volume of the losses diffused across the network and, consequently, with respect to the propensity of a network towards systemic risk. Conclusions are drawn in section five.

\section{The financial flow network}

Let $N$ be a financial system composed of a set of operators $\Omega=\left\{\omega_{i} \mid \mathrm{i}=1, \ldots, \mathrm{n}\right\}$, such as banks and other intermediaries, which are directly or indirectly connected to one another by financial obligations, namely bonds, and let $d_{i j} \in \mathrm{R}^{+}$be the face value of the bond, if any, issued by agent $i$ and held by agent $j$. Each of such operators is characterized by its own balance sheet: $a_{i}+c_{i}=e_{i}+d_{i}+h_{i}$, where: i) $a_{i} \in \mathrm{R}^{+}$is the value of the external assets owned by the $i$-th operator. Such assets are liabilities — shares, bonds and bank loans - of final users of funds, which are not financial operators; ii) $c_{i}=\sum_{j} d_{j i}$ is the sum of the internal assets - which are bonds issued by other agents in $\Omega$ - held by agent $i$; iii) $d_{i}=\sum_{j} d_{i j}$ is the internal debt of agent $i$, i.e., the sum of the liabilities issued by agent $i$ and held by other agents in $\Omega$; iv) $h_{i}$ is the external debt of agent $i$, i.e., the amount of debt claims against $i$ held in the form of bonds and deposits by final claimants, such as households, who do not belong to $\Omega$; and finally v) $e_{i}$ is the value of the equity of the $i$-th agent, which is set residually by the budget identity $e_{i}=a_{i}+$ $c_{i}-d_{i}-h_{i}$. In what follows we assume that there is no cross-holding of shares among the financial intermediaries: all the shares issued by the members of $\Omega$ are held by households.

As in Eboli (2010), we represent the above financial system as a multisource flow network, i.e., a directed, weighted and connected graph, with some sources and two sinks. (Note 2) Let $N=\{\Omega, L, \mathrm{~A}, \mathrm{~T}, \mathrm{H}, \Gamma\}$ be a financial flow network where:

1). $\Omega=\left\{\omega_{i} \mid i=1 \ldots n\right\}$ is the set of $\mathrm{n}$ nodes that represent the above defined financial intermediaries.

2). $L=\left(L^{\Omega} \cup L^{A} \cup L^{T} \cup L^{H}\right)$ is a set of directed links, where i) $L^{\Omega} \subseteq \Omega^{2}$ is a set of ordered pairs of nodes in $\Omega$, i.e., a set of directed links $\left\{l_{i j}\right\}$ representing the liabilities $d_{i j}$, where $l_{i j}$ starts from node $\omega_{i}$ and ends in node $\omega_{j}$, and $l_{i j} \in L^{\Omega}$ only if $d_{i j}>0$; ii) $L^{A}=\left\{l_{i}^{k}\right\}$ is a set of directed links, with start nodes in A and end nodes in $\Omega$, that connect the external assets to their owners, where $l_{i}^{k} \in L^{A}$ only if $a_{i}^{k}>0$; iii) $L^{T}=\left\{l_{i}^{T}\right\}$ is a set of n directed links, with start nodes in $\Omega$ and end node T; and iv) $L^{H}=\left\{l_{i}^{H}\right\}$ is a set of n directed links, with start nodes in $\Omega$ and end node $\mathrm{H}$.

3). $\mathrm{A}=\left\{a_{k} \mid k=1 \ldots m\right\}$, is the set of source nodes, i.e., nodes with no incoming links, that represent the external assets held by the members of $\Omega$.

4). $\mathrm{T}$ is a sink, i.e., a terminal node with no outgoing links. This node represents the shareholders who own the equity of the agents in $\Omega$.

5). $\mathrm{H}$ is a sink node representing the households who hold debt claims, in the form of deposits and bonds, against the agents in $\Omega$.

6). $\Gamma: L \rightarrow \mathbb{R}^{+}$is a map, called capacity function, that associates i) to each $l_{i j}$ the value of the corresponding liability $d_{i j}$, ii) to each $l_{i}^{k}$ the value of the corresponding asset $a_{i}^{k}$, iii) to each $l_{i}^{T}$ the equity, $e_{i}$, of its start node $\omega_{i}$, and iv) to each $l_{i}^{H}$ the external debt, $h_{i}$, of its start node $\omega_{i}$.

A flow in a generic network is a function $g: L \rightarrow \mathbb{R}^{+}$such that: i) for all the links in the network, the scalar associated to a link does not exceed its capacity (capacity constraints); and ii) for all the nodes in the network which are neither a source node nor a terminal node, the divergence - i.e., the difference between the total flow arriving at a node and the total flow departing from such a node - is null (flow conservation property). A flow that complies with these two requirements is said to be legitimate, i.e., it exists.

A flow of value crosses a financial flow network $N$, a flow that starts from the external assets (the sources of value) and ends into the households portfolios (the sink nodes), through the intermediation of the financial operators. If all agents in $\Omega$ are solvent, this value flow is such that all links are filled to capacity (i.e., the value flow coincides with the capacity function $\Gamma$ ). However, since we are interested in measuring direct financial contagion under different accounting regimes, we do not study the properties of such a value flow and rather focus on the flows of losses that cross a financial network $N$ when it is perturbated by a negative exogenous shock. 


\section{Direct contagion with historical cost and mark-to-market accounting rules}

We now proceed to model the domino effect among the agents in $\Omega$, caused by a set of initial defaults, as a flow of financial losses that crosses $N$. This flow is generated by a negative shock, i.e., an exogenous loss of value of some of the external exposures $a_{k}$. Let $b^{k} \in[0,1]$ be a parameter that measures the fraction of the value of the asset $a^{k}$ which is lost, and let $\left[b^{k}\right], k \in A$, be the vector composed of such parameters. An exogenous shock is an assignment of value to this vector, where at least one of its elements is strictly positive. If $b^{k}>0$, then source node $a^{k}$ sends to its children nodes - its direct descendants in $\Omega-$ a financial loss equal to $b^{k} a^{k}$. In other words, a shock is a flow of losses that goes from the nodes in A into the nodes in $\Omega$. The way such a shock propagates among the nodes in $\Omega$ depends on the accounting rules adopted by the financial intermediaries. Eboli (2010b) analyses in details the properties of a propagation function defined over a network $N-$ i.e., the function that associates to an exogenous shock the values taken on by the flows of losses on each of the links in $N$ - with historical cost accounting. For our present purposes, we use two slightly modified versions of the model in Eboli (2010b). In the first version presented below, the one with historical cost accounting, we introduce bankruptcy costs for the sake of realism, (Note 3) such costs include the administrative costs of liquidation and the loss of value arising from the liquidation of firm-specific assets. In the second version, the mark-to-market one, we further modify the model assuming that all the internal assets held by the agents in $\Omega$ are marked at current market prices.

\subsection{Contagion with historical cost accounting}

Let $N$ be a financial flow network and let us assume that the external assets $a_{i}$ of an intermediary in $N$ are accounted at market value, while his other balance sheet headings $c_{i}, d_{i}, h_{i}$, are accounted at their historical cost.

As a shock occurs, some nodes in $\Omega$ suffer a loss. This loss is first offset by the equity of the nodes and borne by their shareholders. These losses exit from the flow of losses that circulates among the agents in $\Omega$ and go directly into the sink T. For each node in $\Omega$, we have an absorption function that measures the share of net worth lost by a node

$$
\beta_{i}\left(\lambda_{i}\right)=\min \left(\frac{\lambda_{i}}{e_{i}}, 1\right)
$$

where $\lambda_{i}$ is the total loss borne by the $i$-th node, received from source nodes and/or from other nodes in $\Omega$. If a node $\omega_{i}$ receives a positive flow of losses, it sends to the sink T an amount of its own equity equal to $\beta_{i} e_{i}, \beta_{i} \in[0,1]$. If the losses suffered by $i$-th intermediary are larger than its net worth, $\lambda_{i}>e_{i}$, then this node is insolvent and sends the loss which is not absorbed by its equity, $\lambda_{i}-e_{i}$, augmented by its bankruptcy costs $\theta_{i}$, to its creditors. For each node in $\Omega$, let

$$
b_{i}\left(\lambda_{i}\right)=\left\{\begin{array}{c}
0, \quad \text { if } \lambda_{i}<e_{i} ; \\
\frac{\lambda_{i}+\theta_{i}-e_{i}}{d_{i}+h_{i}}, \quad \text { if } \lambda_{i} \geq e_{i} .
\end{array}\right\}
$$

be the historical cost loss function. If the $i$-th operator is solvent, $b_{i}$ is null, while $b_{i} \in(0,1]$ if the operator defaults. In the latter case, the assets of the insolvent node are liquidated at current market prices and its creditors get a pro rata refund. Households receive a loss equal to $b_{i} h_{i}$ (if $h_{i}>0$ ), that ends into the sink H, while a node $\omega_{j}$ which is a creditor of node $\omega_{i}$-i.e., a children node $\omega_{j} \in C\left(\omega_{i}\right)=\left\{\omega_{j} \mid \exists l_{i j} \in L\right\}$ of node $\omega_{i}$ - receives from the latter a loss equal to $b_{i} d_{i j}$. The loss borne by a financial intermediary in $\Omega$ is the sum of the losses, if any, received from its external and internal exposures, i.e., form the source nodes and from its parent nodes $\mathrm{P}\left(\omega_{i}\right)=$ $\left\{\omega_{j} \mid \exists l_{j i} \in L\right\}:$

$$
\lambda_{i}=\sum_{\mathrm{k} \in \mathrm{A}} b^{k} a_{i}^{k}+\sum_{j \in \mathrm{P}\left(\omega_{\mathrm{i}}\right)} b_{i} d_{i j}
$$

When a shock perturbates the network, the subsequent flow of losses that crosses the network is governed by the above defined absorption and loss-given-default functions, which assign a positive real value to each link in $N$ given the value taken on by the shock vector $\left[b^{k}\right]$.

Definition 1: Let $f: L \rightarrow R^{+}$be a map such that: $f\left(l_{i}^{k}\right)=b^{k} a_{i}^{k}, f\left(l_{i j}\right)=b_{i} d_{i j}, f\left(l_{i}^{H}\right)=b_{i} h_{i}, f\left(l_{i}^{T}\right)=\beta_{i} e_{i}$, and call this function a historical cost propagation in a network $N$.

This propagation function satisfies the capacity constraints (Note 4) while, for $f$ to be a legitimate flow in $N$, we need to ensure that it complies with the flow conservation property. (Note 5) More precisely, $f$ is an augmenting flow and $N$ is a network with gains, (Note 6) because the default of a node increases its inflow of losses of an amount equal to the incurred bankruptcy costs. The administrative costs of liquidation are extra costs that, in principle, can render the losses borne by an agent larger that the total of its liabilities. In such a case it is unclear who and how 
should pay for such costs. As far as we are here concerned, if $\lambda_{i}+\theta_{i}>e_{i}+d_{i}+h_{i}$, then the inflow of node $i$ is larger than its largest possible outflow and its divergence is strictly positive, violating the flow conservation property. To avoid this formal problem, we assume that, even in the worst scenario, each agent in $\Omega$ owns some residual assets (say properties) and that the value of such assets is sufficient to cover administrative bankruptcy costs:

Assumption 1: $\lambda_{i}+\theta_{i} \leq e_{i}+d_{i}+h_{i}$; for all $i \in \Omega$.

The interdependence of financial obligations that arises in a network $N$, can create problems of non-uniqueness of a propagation. Eboli $(2010$ b) demonstrates that such indeterminacy is confined to closed strongly connected components of defaulting agents, which are subsets $S$ of $N$ such that: i) for each pair of nodes $(i, j)$ in $S$, there exists a directed path going from $i$ to $j$ and a directed path going for $j$ to $i$ ( $S$ is a strongly connected component of $N$ ), ii) no node in $S$ has debts towards nodes in $M S$, and iii) all nodes in $S$ default. In order to ensure the uniqueness of $f$, it is sufficient to assume the following:

Assumption 2: The financial flow network $N$ entails no closed strongly connected components.

This assumption is fairly mild, it implies that for each strongly connected component $S$ in $N$, if any, there is at least one node in $S$ that has a financial obligation towards a node in $\Omega S$ or towards the households in $\mathrm{H}$. Under assumptions 1 and 2, the above defined historical cost propagation $f$ in a network $N$ exists and is unique.

\subsection{Contagion with marking-to-market accounting}

Let us now consider the marking-to-market accounting regime, i.e., we assume that both the internal and the external assets of an intermediary in $\Omega, c_{i}$ and $a_{i}$, are accounted at market value, while its liabilities, $d_{i}$ and $h_{i}$, are accounted for at their historical cost. (Note 7) The market value of a bond embeds its expected loss-given-default. The marking-to-market of a bond, in the balance sheets of its holders, turns such an expected loss into an accounted loss. When the value of the external assets of some agents in $\Omega$ drops, so does the market value of the debt issued by those agents. In compliance with the mark-to-market accounting rule, the holders of such debt claims register this loss in their own balance sheets. As the market value of the internal assets of such debt-holders diminishes, so does the market value of the debt that they issued; this, in turn, inflicts a loss of (internal) assets value to their creditors, and so on and so forth along the chains of obligations in $N$. It is the case to remark that this transmission of losses does not require the actual default of any of the involved agents, it unfolds through the simple weakening of their balance sheets.

To represent this form of contagion, we model the pricing of a defaultable bond as a function of the value of the assets owned by the issuer of such a bond, as it is customary in the structural models of debt pricing based on Merton (1974). (Note 8) For our purposes, all we need is a basic property of such models: the value of a defaultable bond is an increasing, concave and bounded function of the expected value of the issuer's assets. (Note 9)

Let $x_{i}=a_{i}+c_{i}$ be the random variable that measures the market value of the assets owned by agent $i$, let $\pi\left(x_{i}\right)$ be the probability density function of $x_{i}$ and assume, as customary, that the mean and variance of $\pi\left(x_{i}\right)$ are finite. We assume that the market sets the prices with risk-neutral (arbitrage free) probabilities. Under these condition, asset prices are martingales: the current market value of the assets, $x_{i}$, is equal to their expected value: $\bar{x}_{i}=$ $\int_{0}^{\infty} x_{i} \pi\left(x_{i}\right) d x_{i}$; and the market price $p_{i}$ of a bond issued by agent $i$ is equal to its own expected value:

$$
p_{i}\left(x_{i}\right)=\frac{1}{d_{i}+h_{i}} \int_{0}^{d_{i}+h_{i}} x_{i} \pi\left(x_{i}\right) d x_{i}-\frac{\theta_{i}}{d_{i}+h_{i}} \int_{0}^{d_{i}+h_{i}} \pi\left(x_{i}\right) d x_{i}+\frac{1}{d_{i}+h_{i}} \int_{d_{i}+h_{i}}^{\infty} \pi\left(x_{i}\right) d x_{i}
$$

where, for convenience, we set the face value of the bond as equal to unity (hence $p_{i}$ is the price of one unit of the debt issued by agent $i$ ). It can be checked by inspection that $p_{i}\left(x_{i}\right)$ is increasing and concave in the expected value of its argument, $x_{i}$, and bounded above by unity. If node $i$ suffers a loss, due to an external shock, its current asset value $x_{i}$ diminishes, the probability of default of that node increases, the bonds that it issued become more risky and lose some of their market value. Let $x_{i}^{0}$ be the initial value (before the shock) of the assets of agent $i$, and let $\hat{\lambda}_{i}=x_{i}^{0}-x_{i}$ be the loss suffered by node $i$ under the mark-to-market regime. As node $i$ receives a loss, then also the children nodes of node $i$ receive a loss which is equal to i) the price devaluation of $i$ 's debt, as long as agent $i$ is solvent, or ii) to the loss-given-default, if agent $i$ defaults. Thus we define, for each node in $\Omega$, the following mark-to-market loss function:

$$
\hat{b}_{i}\left(\hat{\lambda}_{i}\right)=\left\{\begin{array}{c}
p_{i}\left(x_{i}^{0}\right)-p_{i}\left(x_{i}^{0}-\hat{\lambda}_{i}\right), \quad \text { if } \hat{\lambda}_{i}<e_{i} ; \\
\frac{\hat{\lambda}_{i}+\theta_{i}-e_{i}}{d_{i}+h_{i}}, \quad \text { if } \hat{\lambda}_{i} \geq e_{i} .
\end{array}\right\}
$$


Then the loss borne by a financial intermediary in $\Omega$ is:

$$
\hat{\lambda}_{i}=\sum_{\mathrm{k} \in \mathrm{A}} b^{k} a_{i}^{k}+\sum_{j \in \mathrm{P}\left(\omega_{\mathrm{i}}\right)} \hat{b}_{j} d_{j i}
$$

As above, we define the flows of losses that a node in $\Omega$ sends to its shareholders as the mark-to-market absorption function:

$$
\hat{\beta}_{i}\left(\hat{\lambda}_{i}\right)=\min \left(\frac{\hat{\lambda}_{i}}{e_{i}}, 1\right)
$$

Definition 2: $\quad$ Let $\hat{f}: \mathrm{L} \rightarrow \mathrm{R}^{+}$be a map such that: $\hat{f}\left(l_{i}^{k}\right)=b^{k} a_{i}^{k}, \hat{f}\left(l_{i j}\right)=\hat{b}_{i} d_{i j}, \hat{f}\left(l_{i}^{H}\right)=\hat{b}_{i} h_{i}, f\left(l_{i}^{T}\right)=\hat{\beta}_{i} e_{i}$, and call this function a mark-to-market propagation in a network $N$.

As above, we assume that $\hat{\lambda}_{i}+\theta_{i} \leq e_{i}+d_{i}+h_{i}$, for all $i \in \Omega$, and that $N$ embeds no closed strongly connected component. Under these two assumptions, $\hat{f}$ is a legitimate and uniquely defined flow in $N$. We can now proceed to a comparison of the different systemic implications, in terms of direct contagion, of the two accounting regimes.

\section{Historical cost versus marking-to-market}

Our main finding is that the flow of losses that crosses a network $N$, as a consequence of a shock $\sigma$, under the mark-to-market accounting regime is larger than (or, under a restrictive condition, equal to) the flow of losses generated by the same shock under the historical cost regime. This results stems from the fact that, with historical cost accounting, only the insolvent nodes send losses to their children nodes, while with marking-to-market all nodes that bear a loss, both solvent and insolvent, transmit losses to all their descendant nodes.

The value of a flow in a network is equal to the total flow that goes from the sources to the sinks. For our purposes, we define a stricter condition of inequality between two flows, comparing not just the value of the overall flow that crosses a network but rather the flows carried by each link in $L$.

Definition 3: Let $\phi$ and $\psi$ be two flows in $N$. $\phi$ is link-wise larger than or equal to $\psi$ if the value taken on by $\phi$ on each link in $N$ is larger than or equal to the one taken on by $\psi$; moreover, $\phi$ is said to be strictly link-wise larger than $\psi$ if the strict inequality holds for at least one link in $N$.

Moreover, let $A(\sigma)=\left\{a^{k} \mid b^{k}>0\right\}$ be the set of source nodes activated by the shock $\sigma$, and let $\Theta$ be the risk orbit of $\sigma$, i.e., the set of nodes $\omega_{i} \in \Omega$ such that there exists a directed path (Note 10) in $N$ that starts from $a^{k} \in A(\sigma)$ and ends in $\omega_{i}$. Note that, with mark-to-market accounting, all nodes in $\Theta$ receive a strictly positive loss.

Theorem 1: Let $N$ be a financial flow network; let $\sigma=\left[b^{k}\right]$ be an external shock to $N$; let $f(\sigma)$ and $\hat{f}(\sigma)$ be, respectively, the historical cost and the mark-to-market propagations induced by $\sigma$; and let $\Theta$ be the risk orbit of $\sigma$. Then: i) $\hat{f}(\sigma)$ is link-wise larger than or equal to $f(\sigma)$, and ii) $\hat{f}(\sigma)$ is link-wise equal to $f(\sigma)$ iff $f(\sigma)$ is such that all nodes in $\Theta$ default.

Proof: The value taken on by a propagation induced by a shock $\sigma$ in a network $N$, is characterized computationally through the iterated application of the absorption and loss functions defined above. (Note 11) We establish the first part of above proposition by showing that at each stage of the computation of a propagation, node by node, the values taken on by $\hat{\beta}_{i}\left(\hat{\lambda}_{i}\right)$ and $\hat{b}_{i}\left(\hat{\lambda}_{i}\right)$ are, respectively, larger than or equal to the values taken on by $\beta_{i}\left(\lambda_{i}\right)$ and $b_{i}\left(\lambda_{i}\right)$.

i) Let $\omega_{i} \in \Omega$ receive a positive loss, from the source nodes in $A(\sigma)$ and/or from its parent nodes in $\Omega$. Let us first suppose that $\lambda_{i}=\hat{\lambda}_{i}$, i.e., the loss received by node $i$ is the same under both accounting regimes. In this case: 1$)$ $\hat{\beta}_{i}\left(\hat{\lambda}_{i}\right)=\beta_{i}\left(\lambda_{i}\right)$, and 2) if $\hat{\lambda}_{i}<e_{i}$, then $\hat{b}_{i}\left(\hat{\lambda}_{i}\right)>b_{i}\left(\lambda_{i}\right)$; if $\hat{\lambda}_{i} \geq e_{i}$, then $\hat{b}_{i}\left(\hat{\lambda}_{i}\right)=b_{i}\left(\lambda_{i}\right)$. Thus, for a given loss suffered by node $i$, the outflow of losses transmitted by this node to its descendants is the same under the two accounting regimes if the node defaults; conversely, such an outflow is larger with mark-to-market than with historical cost accounting if node $i$ remains solvent. Let us now remove the assumption that $\lambda_{i}=\hat{\lambda}_{i}$ and recall that $\lambda_{i}=\sum_{k} b^{k} a_{i}^{k}+\sum_{j} b_{j} d_{j i}$, and $\hat{\lambda}_{i}=\sum_{k} b^{k} a_{i}^{k}+\sum_{j} \hat{b}_{j} d_{j i}$. Then, by the definitions of $b_{j}$ and $\hat{b}_{j}, \lambda_{i}=\hat{\lambda}_{i}$ and $\beta_{i}\left(\lambda_{i}\right)=\hat{\beta}_{i}\left(\hat{\lambda}_{i}\right)$ if, for all parent nodes $\omega_{j} \in P\left(\omega_{i}\right)$ of node $i, \lambda_{j} \geq e_{j}$. Conversely, if $0<\lambda_{j}<e_{j}$ for at least one node $\omega_{j} \in P\left(\omega_{i}\right)$, then $\hat{\lambda}_{i}>\lambda_{i}$ and $\hat{\beta}_{i}\left(\hat{\lambda}_{i}\right)>\beta_{i}\left(\lambda_{i}\right)$. In sum, $\hat{\lambda}_{i} \geq \lambda_{i}-$ where the equality holds if and only if no solvent node in $P\left(\omega_{i}\right)$ receives a loss - hence $\hat{\beta}_{i}\left(\hat{\lambda}_{i}\right) \geq \beta_{i}(\lambda i)$. In other words, the positive outflow of a node is the same under the two accounting regimes if the node defaults or bears no losses at all, otherwise $\hat{b}_{i}(\hat{\lambda} i)>b_{i}(\lambda i)$. By the same token, the positive inflow of a node in $\Omega$ is the same, under the two regimes, if none of its solvent parent nodes suffers a loss, otherwise $\hat{\lambda}_{i}>\lambda_{i}$ and, as a consequence, $\hat{\beta}_{i}\left(\hat{\lambda}_{i}\right)>\beta_{i}\left(\lambda_{i}\right)$. This 
reasoning applies to all nodes in $\Omega$, hence $\hat{f}(\sigma)$ and $f(\sigma)$ are such that $\hat{\beta}_{i}\left(\hat{\lambda}_{i}\right) \geq \beta_{i}\left(\lambda_{i}\right)$ and $\hat{b}_{i}\left(\hat{\lambda}_{i}\right) \geq b_{i}\left(\lambda_{i}\right)$ for any $(N, \sigma)$ pair.

ii) The if part of the statement follows from the definitions of $\beta_{i}, \hat{\beta}_{i} b_{i}$ and $\hat{b}_{i}$. To establish the only if part, suppose that a node $\omega_{i} \in \Theta$ is solvent and bears a positive loss. Then' the children nodes of $\omega_{i}$ receive no loss from $\omega_{i}$ with historical cost accounting, while they receive a strictly positive loss with mark-to-market accounting. In other words, if $\omega_{i} \in \Theta$ is solvent and bears a loss, the links in $\left\{l_{i j} \mid j \in C\left(\omega_{i}\right)\right\}$ carry no flow with historical cost rule while they carry a strictly positive flow with marking-to-market.

The fact that the propagation function is larger with marking-to-market than with historical cost rule, has important implications for the robustness of a network: with marking-to-market, a network is more exposed to default contagion, which is defined as follows. Let $\left[b^{k}\right]$ be a shock vector and let $D$ be the set of agents in $\Omega$ who default as a consequence of this shock with historical cost accounting: $D=\left\{\omega_{i} \in \Omega \mid \sum_{k} b^{k} a_{i}^{k}+\sum_{j} b_{j} d_{j i} \geq e_{i}\right\}$, while, with marking-to-market, $\widehat{D}=\left\{\omega_{i} \in \Omega \mid \sum_{k} b^{k} a_{i}^{k}+\sum_{j} \hat{b}_{j} d_{j i} \geq e_{i}\right\}$. Let $D^{\prime}$ be the set of agents that bear an initial shock large enough to cause their default, i.e., $D^{\prime}=\left\{\omega_{i} \in \Omega \mid \sum_{k} b^{k} a_{i}^{k} \geq e_{i}\right\}$, and let $D^{\prime \prime}=D \backslash D^{\prime}$ (with marking-to-market: $\widehat{D}^{\prime \prime}=\widehat{D} \backslash D^{\prime}$ ) be the set of defaulting agents who would be solvent if they had not received losses from their debtors in $\Omega: D^{\prime \prime}=\left\{\omega_{i} \in D \mid \sum_{k} b^{k} a_{i}^{k}<e_{i}\right\}, \widehat{D}^{\prime \prime}=\left\{\omega_{i} \in \widehat{D} \mid \sum_{k} b^{k} a_{i}^{k}<e_{i}\right\}$. There is no default contagion if the set $D^{\prime \prime}$ ( $\widehat{D}^{\prime \prime}$, with mark-to-market) is empty. We call primary the defaults in $D^{\prime}\left(\widehat{D}^{\prime}\right)$ and secondary the ones in $D^{\prime \prime}\left(\widehat{D}^{\prime \prime}\right)$. To compare the effects of the two accounting regimes, we look at two measures of the vulnerability of a network $N$ with respect to default contagion: the scope and the thresholds of contagion.

The scope of the default contagion generated by a shock is the set of defaults induced by such a shock. It is straightforward to see that, because of the link-wise inequality between the propagations $\hat{f}(\sigma)$ and $f(\sigma)$, the nodes that default with historical cost accounting, also default under mark-to-market accounting, while the converse is not always true:

Corollary 1: Let $N$ be a financial flow network and let $\sigma$ be an external shock to $N$. Let $D(N, \sigma)$ and $\widehat{D}(N, \sigma)$ be the sets of defaulting nodes induced in $N$ by $f(\sigma)$ and $\hat{f}(\sigma)$, respectively. Then $D(N, \sigma) \subseteq \widehat{D}(N, \sigma)$.

Proof : It follows from theorem 1. Since $b_{j}\left(\lambda_{j}\right) \leq \hat{b}_{j}\left(\hat{\lambda}_{j}\right), \lambda_{i} \leq \hat{\lambda}_{i}$ for all $i \in \Omega$, hence $\left\{\omega_{i} \in \Omega \mid \lambda_{i} \geq e_{i}\right\} \subseteq$ $\left\{\omega_{i} \in \Omega \mid \hat{\lambda}_{i} \geq e_{i}\right\}$.

A second measure of the exposure of a network $N$ to systemic risk is the threshold of contagion, (Note 12) which is the minimum value that a shock must reach in order to induce secondary defaults, where the value of a shock $\sigma$ is the sum of the flow out of the source nodes: $v(\sigma)=\sum_{k \in A(\sigma)} b^{k} a^{k}$.

Definition 4: The first threshold of contagion of a financial flow network $N$ is the value of the smallest shock that is large enough to cause secondary defaults. Correspondingly, the final threshold of contagion of a network is the value of the smallest shock that is capable of inducing the failure of all nodes in the network.

Formally, let $\Sigma^{\prime}=\left\{\sigma \mid \lambda_{i} \geq e_{i}\right.$ for some $\left.\omega_{i} \in D^{\prime \prime}\right\}$ be the set of shocks which are large enough to cause default contagion in $N$ with historical cost accounting. Let $\sigma^{\prime}$ be a smallest element of $\Sigma^{\prime}$, then $v\left(\sigma^{\prime}\right)$ is the first threshold of $N$ with historical cost rule. Let $\Sigma^{\prime \prime}=\left\{\sigma \mid \lambda_{i} \geq e_{i}\right.$ for all $\left.\omega_{i} \in D^{\prime \prime}\right\}$ be the set of shocks which are large enough to cause the default of all nodes in $\Omega$ with historical cost accounting. Let $\sigma^{\prime \prime}$ be a smallest element of $\Sigma^{\prime \prime}$, hence $v\left(\sigma^{\prime \prime}\right)$ is the final threshold of $N$ with historical cost accounting. Correspondingly, let $\hat{\Sigma}^{\prime}=\left\{\sigma \mid \hat{\lambda}_{i} \geq\right.$ $e_{i}$ for some $\left.\omega_{i} \in \widehat{D}^{\prime \prime}\right\}$ and $\Sigma^{\prime \prime}=\left\{\sigma \mid \hat{\lambda} \geq e_{i}\right.$ for all $\left.\omega_{i} \in \widehat{D}^{\prime \prime}\right\}$ be the sets analogous to $\Sigma^{\prime}$ and $\Sigma^{\prime \prime}$ with mark-to-market accounting, and let $\hat{\sigma}^{\prime}$ and $\hat{\sigma}^{\prime \prime}$ be their minimal elements, respectively.

Corollary 2: Under a marking-to-market regime, both the first and the final thresholds of contagion of a network $N$ are smaller than, or equal to, the same thresholds under a historical cost regime: $v\left(\hat{\sigma}^{\prime}\right) \leq v\left(\sigma^{\prime}\right)$ and $v\left(\hat{\sigma}^{\prime \prime}\right) \leq$ $v\left(\sigma^{\prime \prime}\right)$.

Proof: Since $\hat{f}\left(\sigma^{\prime}\right)$ is link-wise larger than or equal to $f\left(\sigma^{\prime}\right)$, then $\sigma^{\prime} \in \hat{\Sigma}^{\prime}$, i.e., since $\sigma^{\prime}$ is sufficiently large to cause secondary defaults with historical cost rule, then it is a fortiori sufficient to induce contagion with marking-to-market. Hence, by the definitions of $\hat{\Sigma}^{\prime}$ and $\hat{\sigma}^{\prime}$, we have that $v\left(\hat{\sigma}^{\prime}\right) \leq v\left(\sigma^{\prime}\right)$. This relation is a weak inequality (rather than an equality) because $\hat{f}\left(\sigma^{\prime}\right)$ is link-wise larger than or equal to $f\left(\sigma^{\prime}\right)$, hence $\hat{\sigma}^{\prime}$ is not necessarily in $\Sigma^{\prime}$. The same reasoning applies to the final thresholds: $\sigma^{\prime \prime} \in \hat{\Sigma}^{\prime \prime}$, while $\hat{\sigma}^{\prime \prime}$ is not necessarily in $\Sigma^{\prime \prime}$.

Finally, the residual capitalization of all solvent nodes in the risk orbit $\Theta$, is larger with the historical cost propagation than with the mark-to-market propagation, unless all nodes in $\Theta$ default. 
Corollary 3: Let $N$ be a financial flow network, let $\sigma$ be an external shock to $N$ and let $e_{i}^{*}$ be the residual equity of node $\omega_{i} \in \Omega$ posterior to the propagation with the historical cost accounting regime $f(\sigma)$, and let $\hat{e}_{i}^{*}$ be the residual equity of node $\omega_{i} \in \Omega$ posterior to the propagation with the marking-to-market regime $\hat{f}(\sigma)$. Then $e_{i}^{*}>\hat{e}_{i}^{*}$ for all solvent nodes in the risk orbit $\Theta$ of $\sigma$.

Proof: The proof is trivial and is omitted.

In sum, the marking-to-market of assets, compared to the historical cost regime, exacerbates the systemic effects of external shocks, increasing the vulnerability of financial networks like $N$.

\section{Conclusions}

The rationale for the adoption of the fair value accounting regime is the enhancement of market transparency. According to the Securities and Exchange Commission (2008), marking-to-market provides "the most transparent financial reporting of an investment, thereby facilitating better investment decision-making and more efficient capital allocation". But while, on one hand, this accounting regime improves the information available to market participants, on the other hand it has undesirable systemic implications in times of financial turbulence. When the markets for illiquid assets are affected by liquidity pricing, the marking-to-market of such assets becomes a vehicle of financial contagion, as argued by the above cited works by Shin et al. (2005) and Allen and Carletti (2007). In this paper we analyzed another source of systemic effects of marking-to-market: the strengthening of the direct balance sheet contagion that unfolds, among financial intermediaries, through their cross-holding of debt. With marking-to-market, the transmission of losses from debt-issuers to debt-holders does not require the occurrence of defaults, for this accounting rule turns the expected losses, embedded in market prices, into accounted losses. Conversely, with accounting at historical cost, the transmission of losses from an agent towards his creditors occurs only in case of default. Because of this simple difference, the flow of losses that crosses a financial network, induced by an exogenous negative shock, is larger with marking-to-market than with historical cost accounting. As a consequence, a financial network is more exposed to systemic risk with mark-to-market than with historical cost accounting. We showed that the propagation of losses generated by an exogenous shock with the marking-to-market rule, compared to the historical cost regime, has the following disadvantages: i) induces a larger set of defaults, ii) sets smaller thresholds of default contagion (i.e., renders a financial network vulnerable to smaller shocks), and iii) leaves a smaller residual equity to the surviving agents.

\section{References}

Ahuja, K. R., Magnanti, T. L. and Orlin, J. B. (1993). Network Flows: theory, algorithms and applications, Prentice Hall, New Jersey.

Allen, F. and Carletti, E. (2008). "Mark-to-market accounting and liquidity pricing", Journal of Accounting and Economics, 45 (2-3)., 358-378.

Brealey, R. A. and Myers, S. C. (2002). Principles of Corporate Finance, international edition, McGraw-Hill.

Dirikxs, I. M. and Rao, R. M. (1974). "Networks with gains in discrete dynamic programming", Management Science, vol. 20, no11.

Eboli, M. (2010 a). "An algorithm of propagation in weighted directed graphs with applications to economics and finance", International Journal of Intelligent Systems, Volume 25 Issue 3, p. 237-252.

Eboli, M. (2010 b). "The mechanics of direct contagion in financial networks: a graph-theoretic approach", mimeo, available from the author.

Freixas, X. and Tsomocos, D. P. (2006). "Book vs. Fair Value Accounting in Banking, and Intertemporal Smoothing", available on-line at: http://www.eu-financial-system.org/index.php?id=81.

Jensen, P. A. and Bhaumik, G. (1977). "A Flow Augmentation Approach to the Network with Gains Minimum Cost Flow Problem", Management Science, vol. 23, nº 6 .

Merton, R. C. (1974). "On the pricing of corporate debt: The risk structure of interest rates", Journal of Finance, 29, 449-470.

Securities and Exchange Commission. (2008). Report and recommendations pursuant to section 133 of the Emergency Economic Stabilization Act of 2008: study on mark-to-market accounting. Available online at: www.sec.gov/news/studies/2008/marktomarket123008.pdf.

Shin, H. S., Cifuentes, R., and Ferrucci, G. (2005). "Liquidity risk and contagion", Journal of European Economic Association, 3, 556-566. 
Shin, H. S., Sapra, H. and Plantin, G. (2008). "Marking to Market: Panacea or Pandora's Box?", Journal of Accounting Research, 46, $435-460$.

\section{Notes}

Note 1. See Plantin-Sapra-Shin (2008) for an evaluation of the pros and cons of marking-to-market vs historical cost in presence of i) liquidity pricing of illiquid assets, and ii) short-termism of managerial incentive schemes. As the authors put it: "The historical cost regime relies on past transaction prices, and so accounting values are insensitive to more recent price signals. This lack of sensitivity to price signals induces inefficient sales because the measurement regime does not reflect the appreciated value of the measured assets. Marking-to-market overcomes this price insensitivity by extracting the information conveyed by market prices, but it also distorts the information for illiquid assets [...]." [Shin et al. (2008), page 438]. See also Freixas-Tsomocos (2006), who study the implications of fair vs. book value accounting practice for the banking sector efficiency in providing intertemporal wealth transfer.

Note 2. See Ahuya et al. (1993) for a comprehensive treatment of flow networks.

Note 3. In absence of bankruptcy costs, the below defined price function of a bond would display an implausible jump in case of default of the issuer.

Note 4. Since $b_{i}$ and $\beta_{i}$ are all smaller than or equal to unity.

Note 5 Existence and uniqueness of a propagation function in a financial flow network are discussed in details in Eboli (2010b).

Note 6. See, inter alia, Jensen and Bhaumik (1977) and Dirikxs and Rao (1974) for applications of augmenting flows in network with gains. Formally, we should add to $N$ extra source nodes, one for each node in $\Omega$, that get activated in case of default and release a loss equal to the bankruptcy costs. This technicality is here redundant and is omitted.

Note 7. This amounts to assume that all liabilities are held to maturity, i.e., no agent in $\Omega$ undertakes a buy-back of its own bonds.

Note 8. A large literature on debt pricing has developed, starting with the work by Merton (1974) who introduced the use of option pricing models for assessing the value of a defaultable bond, pointing out its equivalence with a portfolio composed of an amount of cash equal to the face value of the bond and a short position on a put option on the assets of the issuer, with strike price equal to the face value of the bond. Successively, several authors have extended the model by Merton in various directions. Such models, known as structural models, assess default probabilities, hence bonds prices, by modeling the stochastic processes that drive the value of the assets of the issuers. Conversely, a more recent class of models, known as reduced from models, take such default probabilities as exogenously determined, rather than deriving them from asset values.

Note 9. See, inter alia, Brealey and Myers (2002), sections 22-2 and 23-3

Note 10. A directed path (also known as a walk) in a directed graph is an alternating sequence of nodes and links, where each node is connected to the successive node in the sequence by a directed link.

Note 11. See Eboli $(2010 \mathrm{a}, \mathrm{b})$ for two algorithms, designed to compute a propagation in a flow network $N$, based on the iterated application of such absorption and loss functions.

Note 12. Eboli (2010b) characterizes and compares first and final thresholds of default contagion of differently shaped networks, such as complete, incomplete, star-shaped and chain-shaped networks. 